\title{
BMJ Open Use of inhalational anaesthetic agents in paediatric and adult patients for status asthmaticus, status epilepticus and difficult sedation scenarios: a protocol for a systematic review
}

\author{
Kevin Gorsky (1) , ${ }^{1}$ Sean Cuninghame (D) , ${ }^{2}$ Jennifer Chen, ${ }^{3}$ Kesikan Jayaraj, ${ }^{4}$ \\ Davinia Withington, ${ }^{5}$ Conall Francoeur, ${ }^{6}$ Marat Slessarev, ${ }^{2,7}$ Angela Jerath ${ }^{1,8}$
}

To cite: Gorsky K, Cuninghame S, Chen J, et al. Use of inhalational anaesthetic agents in paediatric and adult patients for status asthmaticus, status epilepticus and difficult sedation scenarios: a protocol for a systematic review. BMJ Open 2021;11:e051745. doi:10.1136/ bmjopen-2021-051745

- Prepublication history and additional supplemental material for this paper are available online. To view these files, please visit the journal online (http://dx.doi.org/10.1136/ bmjopen-2021-051745).

Received 26 March 2021 Accepted 20 October 2021

Check for updates

(C) Author(s) (or their employer(s)) 2021. Re-use permitted under CC BY-NC. No commercial re-use. See rights and permissions. Published by BMJ.

For numbered affiliations see end of article.

Correspondence to

Dr Kevin Gorsky;

kevin.gorsky@mail.utoronto.ca

\section{ABSTRACT}

Introduction Inhaled volatile anaesthetics have a long tradition of use as hypnotic agents in operating rooms and are gaining traction as sedatives in intensive care units (ICUs). However, uptake is impeded by low familiarity with volatiles, unique equipment and education needs. Inhaled anaesthetics are often reserved in ICUs as therapies for refractory and life threatening status asthmaticus, status epilepticus, high and difficult sedation need scenarios given they possess unique pharmacological properties to manage these medical conditions while providing sedation to acutely ill patients. The objective of this systematic review is to collate evidence regarding the efficacy, safety and feasibility of volatile anaesthetics in adult and paediatric ICU patients for these three emergency conditions.

Methods and analysis We will conduct a systematic review of the primary studies in adult and paediatric ICU patients with status asthmaticus, status epilepticus and high/difficult sedation needs. We will include observational and interventional studies published from 1970 to 2021 in English or French investigating patients who have received a volatile inhalational agent for the above indications. We will evaluate the efficacy, safety, feasibility and implementation barriers for the volatile anaesthetics for each of three specified indications. Included studies will not be limited by necessity of a comparator arm. We will also evaluate clinical characteristics, patient demographics and provider attitudes towards volatile anaesthetic administration in defined critical care scenarios. Data will be extracted and analysed across these domains. The databases MEDLINE, EMBASE, the Science Citation Index as well as the Cochrane Central Controlled Trials Register will be queried with our search strategy.

Descriptive and statistical analysis will be employed where appropriate. Data extraction and quality assessment will be performed in duplicate using a standardised tool. A narrative approach and statistical analyses will be used to describe patient characteristics, volatile efficacy, safety concerns, technical administration, attitudes towards administration and other implementation barriers. Ethics and dissemination No ethics board approval will be necessary for this systematic review. This research is
Strengths and limitations of this study

- Low quality evidence (case series and reports) will inform the majority of included studies.

- Expansive inclusion criteria will capture a large number of appropriate studies.

- Electronic database search is appropriately thorough for the research question.

- Multiple tiers of study review will ensure quality and accuracy of data.

- Study design and data extraction tools are optimised for qualitative and descriptive statistical analysis.

independently funded. Results will be disseminated in a peer-reviewed journal and conference presentation. PROSPERO number CRD42021233083.

\section{INTRODUCTION}

Background

Volatile agents are ether-based compounds with general anaesthetic properties that are conventionally delivered via inhalation. The most commonly used inhalational agents in modern medical practice include isoflurane, sevoflurane, desflurane and nitrous oxide. ${ }^{1}$ The nomenclature inhalational agents captures both volatile anaesthetics, as well as other gaseous compounds such as nitrous oxide. These drugs have a long tradition of safe and effective use in operating room environments, providing deep hypnosis and amnesia appropriate for surgical levels of anaesthesia. ${ }^{1}$

Inhalational agents are being used with increasing frequency within intensive care units (ICU) to provide sedation, which is required by most patients needing tracheal intubation and mechanical ventilation. ${ }^{23}$ Volatile inhalational agents possess rapid onset and offset of clinical effect, and have been 
shown to promote faster patient awakening-particularly in the postoperative setting. ${ }^{24}$ Widespread use of inhaled sedatives has been limited by lack of familiarity with this drug group, specific equipment systems and need for trained personnel to deliver these agents. Although there have been technical advancements to simplify volatile delivery in ICU settings, most physicians use inhalational agents as 'rescue' therapies in life-threatening medical emergencies that are unresponsive to standard medical therapies. These include status asthmaticus, status epilepticus and patients with high/difficult sedation needs (eg, burns, chronic pain, illicit drug use).

\section{Rationale}

Status asthmaticus is a severe form of asthma exacerbation that is characterised by hypoxaemia, hypercarbia and progressive respiratory failure. Status asthmaticus patients show poor response to standard medical therapies used to treat bronchospasm, airway inflammation and improve gas exchange such as beta 2-agonists, anticholinergics, methylxanthines, ketamine and steroids. ${ }^{5}$ Status asthmaticus affects between $3 \%$ and $16 \%$ of hospitalised adult asthmatic patients (with lower incidence in children) and requires ICU transfer and mechanical ventilation to manage severe respiratory failure. ${ }^{6}$ Inhaled volatile agents are used as rescue therapies to manage lifethreatening ventilated status patients given they possess unique bronchodilator properties that can break severe bronchospasm and improve gas exchange, while also providing sedation. The full mechanism by which volatile agents relax airway smooth muscle is incompletely understood. In vitro studies, however, suggest volatiles reduce intracellular free calcium via the inhibition of protein kinase C, calcium release from sarcoplasmic reticulum, and voltage-dependent calcium channels, which leads to relaxation of the bronchial smooth muscle. ${ }^{5}$ Volatile anaesthetics also relax the bronchial muscle tree by reducing vagal tone, altering circulating catecholamine and beta receptor sensitivity. ${ }^{5}$ Volatile anaesthetics directly act on bronchial smooth muscle by diffusion across the airway wall and systemically by entering the bronchial and pulmonary circulations.

Status epilepticus (SE) is defined as seizure with more than 5 min of continuous clinical and/or electrographic seizure activity, or recurrent seizure activity without recovery between seizures. Seizure activity may be convulsive, non-convulsive, focal motor or myoclonic. ${ }^{7}$ The incidence of status epilepticus ranges between 7 and 40 cases per $100000 .{ }^{78}$ Status epilepticus is most common among males, and peaks in both infancy and older age. The mechanisms underlying status epilepticus are multifactorial and include changes in the inhibitory gamma aminobutyric acid-A (GABA-A) receptors with reduced responsiveness to GABA-mediated medications such as benzodiazepines. ${ }^{8-10}$ Status epilepticus patients display upregulation of p-glycoprotein that causes rapid export of phenytoin and phenobarbital molecules leading to poor action of these traditional antiepileptic drugs. ${ }^{8}$ In addition, prolonged seizure activity leads to upregulation of N-methyl D-aspartate receptors and glutamate mediated excitotoxicity, further potentiating epileptic activity. ${ }^{8}$ Refractory status epilepticus refers to ongoing seizure activity that continues after first-line and secondline therapy has failed, although the duration of seizures and number of trialled antiepilepticus varies between definitions. ${ }^{11-13}$ Refractory disease occurs in 9\%-40\% of status epilepticus patients and represents a medical emergency with a $50 \%$ mortality rate and significant morbidity. ${ }^{12}{ }^{14}$ Volatile inhalational agents provide deep levels of sedation and are powerful anticonvulsants that are reserved for patients with seizures that are refractory to traditional intravenous medications such as benzodiazepines, propofol, barbiturates and standard antiepileptic drugs. Inhaled volatile agents provide anticonvulsant effects by promoting central inhibitory GABAa pathways and lowering activity of excitatory NMDA pathways. ${ }^{15} 16$

Inhaled anaesthetic agents are used for sedation in ICU patients with prolonged and difficult sedation needs. In these patients, inhaled volatiles provide several distinct advantages over traditional intravenous ICU sedatives (ie, benzodiazepines, propofol, opioids, ketamine) including continuous gas monitoring that facilitates better dose titration with reliable levels of sedation. ${ }^{17}{ }^{18}$ Inhalational agents are also less likely to show ceiling effects and tolerance, issues seen with prolonged use and high doses of intravenous sedatives and opioids. ${ }^{2}$ In contrast to intravenous sedatives that depend on hepatorenal metabolism, inhaled volatile agents are cleared through pulmonary exhalation with no systemic drug accumulation. This property minimises the effect of prolonged drug clearance that can slow patient recovery of consciousness from high systemic drug and active metabolite concentration levels. There is growing evidence demonstrating the utility of inhaled volatile anaesthetics as ICU sedatives with faster emergence and extubation properties when compared with intravenous agents in adult populations. ${ }^{2}$ These properties are used to rescue patients with complex and high sedation needs (ie, illicit drug use, chronic pain with high and complex analgesic requirements) or patients where there are concerns of sedative drug and metabolite accumulation impacting patient outcomes.

There is limited study evaluating the use, safety and feasibility of delivering inhaled volatile agents in adult and paediatric ICUs. Optimal dosing, delivery systems and technical challenges associated with their use have not, to our knowledge, been investigated within the above three clinical scenarios. Postoperative sedation from adult trials using inhaled volatile for less than 24 hours show that these agents have a similar safety profile to intravenous sedatives (ie, dose-dependent haemodynamic instability, respiratory depression, hypnosis, idiosyncratic reactions such drug induced nephrogenic diabetes insipidus). ${ }^{19}$ Evaluation of adverse effects during prolonged therapy within these three medical emergencies has not been well described. 
Inhaled volatile anaesthetics are not a panacea; like all sedatives used in critical care, they have been associated with various adverse events and rare side effects when administered for both the short and long term. This includes common issues of dose-dependent hypotension, raised intracranial pressure, arrhythmias as well as rare serious complications of malignant hyperthermia. ${ }^{2} 20$ Nitrous oxide can be associated with expansion of gas spaces for example, enlargement of a pneumothorax or ileus, and can produce irreversible oxidation of the cobalt component of vitamin $B_{12}$ which may lead to megaloblastic anaemia and subacute combined degeneration of the spinal cord in vulnerable critically ill patients. ${ }^{21}$ Moreover, there is a concern for occupational exposure when using inhalational anaesthetics if proper scavenging is not employed. Importantly, research has demonstrated the safe use of volatiles in the ICU setting without significant bedside contamination when appropriate equipment is used. ${ }^{20}$ Further, these agents leave a significant environmental footprint with considerable green house gas effects in the atmosphere. ${ }^{20}$

There is a clear biological and mechanistic rationale supporting the use of inhaled volatile agents for managing status asthmaticus, status epilepticus and patients with high sedation needs. However, these agents are typically used as 'rescue' therapies, in part due to poor understanding of their pharmacology and unfamiliarity with inhaled delivery systems required for their delivery. Miniature vaporiser systems that integrate with existing ICU ventilators have simplified delivery of these agents in ICU settings. This has enabled several small clinical trials assessing the efficacy of volatiles as primary ICU sedatives for short duration postoperative sedation and longerterm sedation in adult general medical-surgical ICU patients. ${ }^{49}{ }^{20}$ Whether inhaled volatile agents should be employed as first line therapy for their combined sedative and therapeutic properties for severe asthma, seizures and high sedation users has never been evaluated. Prior to undertaking a trial in any of these medical conditions, this review aims to collate evidence regarding the efficacy, safety and feasibility of their use in ventilated adult and paediatric ICU patients.

\section{Objectives}

The objective of this systematic review is to evaluate the safety, efficacy and feasibility of inhaled volatile agents in paediatric and adult ICU patients with status epilepticus, status asthmaticus or high/difficult sedation requirements.

This systematic review aims to answer the following research questions:

1. What is the efficacy of using inhaled volatile agents for the above three medical indications in achieving (1) resolution of refractory seizure activity in status epilepticus, (2) resolution of refractory bronchospasm in status asthmaticus or (3) achieving targeted sedation outcomes in patients with high and/or difficult sedation requirements.
2. What are the short-term and/or long-term adverse events related to the use of inhaled volatile agents?

3 . What equipment and personnel where required to deliver inhaled volatile agents for the above indications and what issues have been identified?

\section{METHODOLOGY}

This protocol has been developed as per the Preferred Reporting Items for Systematic Review and Meta-Analysis Protocols (PRISMA-P) (online supplemental file 1).

\section{Study eligibility criteria}

This systematic review will look at all published studies (not limited by study design) examining adult and paediatric patients, receiving inhalational anaesthetic agents for one of three specific indications, not limited by necessity of a comparator arm and evaluating composite outcomes including implementation, safety, efficacy and provider attitudes towards inhalational anaesthetic administration in defined critical care scenarios. We will review all studies published after 1970 in either English or French.

\section{Inclusion criteria}

(1) Adult ( $\geq 18$ years) or paediatric $(<18$ years) ventilated ICU patients; (2) Studies will not be limited by study design: We expect the body of literature to consist mainly of case reports and case series. All available observational studies and randomised clinical trials will be assessed. We will cross-reference with previous systematic reviews to ensure we capture important articles; (3) All general and specialised ICUs (cardiac, burns, neurosurgical, trauma) will be included; (4) There is no restriction to sample size and (4) Papers published in English and French 1970 to 2021.

\section{Exclusion criteria}

(1) Studies where the indication for inhaled volatile anaesthetic is not clearly outlined; (2) Patients whereby inhalational sedation was initiated prior to explicit clinical inclusion criteria (ie, postoperative patients who were maintained on volatile after surgery including cardiac surgery); (3) Abstracts; (4) Editorials that do not present new data; (5) Studies examining inhaled anaesthetics agents that are not currently used in modern clinical practice (ie, diethyl ether, xenon, chloroform, ethyl chloride, cyclopropane) and (6) Patients for whom volatile therapy was used for cardioprotection applications and investigations.

\section{Types of participants}

Ventilated adult and paediatric ICU patients requiring inhaled volatile agents for (1) status asthmaticus and refractory bronchospasm, (2) status epilepticus and refractory myoclonic activity/movement disorders and (3) difficult sedation scenario as defined as: (A) difficulty weaning sedation, (B) expected prolonged sedation $>24$ hours with switch to, or initial administration of inhaled volatile anaesthetic and (C) concern of intravenous 
sedation metabolite build up or adverse effects thereof. Studies under the difficult sedation group will not be limited by underlying patient diagnosis or pathology.

\section{Types of exposure/interventions}

Patients within this group will have received volatile/ inhalational sedation using halothane, enflurane, isoflurane, sevoflurane, desflurane and/or nitrous oxide for one of the above clinical indications. We will document the concentration of these agents (if provided), the length and timing of the intervention, the equipment by which the volatile was administered and any additional personnel requirements.

\section{Types of comparator}

We do not anticipate many randomised controlled trials in this area, given inhaled volatiles are used when there is failure of standard medical therapy and the low frequency of the above three emergency medical scenarios.

\section{Outcomes}

Primary outcomes of this systematic review will include data on the efficacy of inhalational volatile agents in three defined clinical scenarios:

\section{Assessing efficacy of inhaled volatile use}

Efficacy of inhaled volatile anaesthetic for the treatment of each specific indication:

1. Status asthmaticus/refractory bronchospasmEfficacy of inhalational volatile agents at breaking bronchospasm (ie, clinical and physical exam improvements, decreased wheeze on auscultation, improved air entry and/or features of improved compliance with ventilation), improving oxygenation and ventilation parameters, improving lung mechanics, de-escalation of medical therapies and weaning from the ventilator (ie, reduction in driving pressures for appropriate ventilation).

2. Status epilepticus and refractory movement disorders-Efficacy of inhalational volatile agents for terminating seizure activity as defined by cessation of epileptiform activity, burst suppression on EEG and/ or clinical status and de-escalation of treatment/antiepileptic adjuncts.

3. Difficult sedation-Efficacy of inhalational sedation for achieving adequate sedation or target sedation score (ie, Ramsey sedation score, Riker sedation score, etc or for paediatric populations COMFORT, State Behaviour Scale, etc), and weaning of other intravenous sedative agents.

Secondary outcomes of this review will include data on the clinical characteristics, safety and feasibility of implementing inhalational anaesthetics for the aforementioned indications:

Clinical characteristics of inhaled volatile implementation

(1) Patient demographics including age, sex and severity of illness score (eg, Sequential Organ Failure Assessment (SOFA), Program of Research on Integration of Services for the Maintenance of Autonomy (PRISMA)), (2) therapies/adjunctive medications used prior to initiation of volatile anaesthetic, time to initiate inhaled volatile therapy, de-escalation of adjuncts with initiation of volatile therapy, (3) duration of inhaled volatile use, ICU length of stay (LOS), ventilation and postvolatile ICU care, (4) concentration of inhaled volatile agent and/or minimum alveolar concentration(MAC)/MAC hours if available and (5) ICU mortality.

\section{Assessing safety of inhaled volatile use}

1. Short-term outcomes during ICU stay-Cardiovascular events (eg, arrhythmias), haemodynamic stability (eg, blood pressure, need for vasoactive drug support), ventilation/gas exchange, neurocognitive changes (eg, seizures, delirium) and neurodegenerative changes (eg, neuroimaging changes), hepatorenal (eg, hepatitis, acute kidney injury (AKI), fluoride levels) and other systemic adverse effects (eg, haematological, metabolic, neurohormonal, etc).

2. Long-term outcomes after hospital dischargeNeuropsychiatric disorders, neurocognitive development.

Assessing feasibility and barriers to successful inhaled volatile implementation

1. Method of inhaled volatile delivery-equipment and monitoring used, any technical concerns.

2. Additional personnel required to deliver inhaled volatile agents.

3. Attitudes towards inhalational agent use among ICU practitioners.

4. Existence of institutional protocol for inhaled volatile anaesthetic use in the ICU.

\section{Electronic search}

A systematic search of all relevant literature in the English and French language will be conducted using electronic databases currently available at the University of Toronto. We plan to make use of MEDLINE, EMBASE and the Science Citation Index as well as the Cochrane Central Controlled Trials Register. A time limit of 1970 to June 2021 will be used. Abstracts will also be reviewed for consideration, only if full text articles can be identified. A sample search strategy for Embase is provided (figure 1).

\section{Other resources}

We will review the reference list of identified studies and review articles to determine if any relevant studies have been missed. Identified abstract will be reviewed for additional consideration only if full text articles can be located.

\section{Data management}

Studies identified from our database query will be collated onto an online resource manager (www.covidence.organ online literature review tool recommended by the Cochrane Collaboration) after duplicates are removed. This online tool will be used for all tiers of the study 
Database: Embase Classic+Embase <1970 to 2021 July 1>

Search Strategy:

1 - exp *inhalation anesthetic agent/ or exp inhalation anesthesia/ (72525)

2 - (( (inhal ${ }^{*}$ or insufflation $\$$ or gas or gaseous $\$$ or volatile $\$$ or halogen $\$$ ) adj3 (an?esthe* or sedat a $\left.^{*}\right)$ or (inhalation\$ adj3 induction $\$$ ) or (ftorotan $\$$ or fluothane $\$$ or narcotan $\$$ or isoflurane $\$$ or aerane $\$$ or aerrane $\$$ or forane $\$$ or forene $\$$ or forthane $\$$ or isoflurano $\$$ or isorane $\$$ or sofloran $\$$ or sevocalm $\$$ or sevoflo $\$$ or sevofrane $\$$ or sevohale $\$$ or sevoflurane $\$$ or sevorane $\$$ or sevotec $\$$ or ultane $\$$ or anestane $\$$ or bromochlorotrifluorethane $\$$ or (bromo $\$$ adj2 chloro $\$$ adj2 trifluorethane $\$$ ) or fluorothan* or halan $\$$ or halothan* or halthane $\$$ or ineltano $\$$ or phthorothane $\$$ or trothane $\$$ or desflurane $\$$ or enflurane $\$$ or ether $\$$ or methoxyflurane $\$$ or penthrox $\$$ or (nitrous $\$$ adj2 oxide $\$$ ) or (nitrogen $\$$ adj2 protoxide $\$$ ) or (laughing $\$$ adj2 gas $\$$ ) or trichloroethylene $\$$ or xenon $\$$ )).tw,kw. (175917)

3 - or/1-2 [volatile anesthetics concept] (201607)

4 - critically ill patient/ or exp intensive care unit/ or intensive care/ or critical illness/ (343219)

5 - ((intens ${ }^{*}$ or critical $\left.{ }^{*}\right)$ adj3 (care or ill or illness or unit*)).tw,kw. (314673)

6 - (ICU or ICUs or CCU or CCUs or ITU or HDU).tw,kw. (130156)

7 - or/4-6 [ICU concept] (472832)

$8-3$ and 7 (2890)

9 - asthmatic state/ or (asthmatic ${ }^{*}$ adj2 (crises or crisis or shock* or status)).tw,kw. (3807)

10 - epileptic state/ or (( (epileptic\$ or absence or petit-mal) adj2 status) or ((continuous $\$$ or prolonged\$ or prolongated\$ or incontrollable $\$$ ) adj5 (epilep\$ adj10 seizure $\$)$ ) or ((epilepsy or syndrome $\$$ ) adj3 (kojevniko $\$$ or kosherniko\$ or kojewniko\$))).tw,kw. (28383)

11 - or/9-10 (32141)

$12-3$ and 11 (390)

13 - (((inhal ${ }^{*}$ or insufflation $\$$ or gas or gaseous $\$$ or volatile $\$$ or halogen $\$$ ) adj3 (an?esthe or sedat $\left.^{*}\right)$ ) or (inhalation\$ adj3 induction $\$$ ) or (ftorotan $\$$ or fluothane $\$$ or narcotan $\$$ or isoflurane $\$$ or aerane $\$$ or aerrane $\$$ or forane $\$$ or forene $\$$ or forthane $\$$ or isoflurano $\$$ or isorane $\$$ or sofloran $\$$ or sevocalm $\$$ or sevoflo $\$$ or sevofrane $\$$ or sevohale $\$$ or sevoflurane $\$$ or sevorane $\$$ or sevotec $\$$ or ultane $\$$ or anestane $\$$ or bromochlorotrifluorethane $\$$ or (bromo $\$$ adj2 chloro $\$$ adj2 trifluorethane $\$$ ) or fluorothan* or halan $\$$ or halothan* or halthane $\$$ or ineltano $\$$ or phthorothane $\$$ or trothane $\$$ or desflurane $\$$ or enflurane $\$$ or ether $\$$ or methoxyflurane $\$$ or penthrox $\$$ or (nitrous $\$$ adj2 oxide $\$$ ) or (nitrogen $\$$ adj2 protoxide $\$$ ) or (laughing $\$$ adj2 gas $\$$ ) or trichloroethylene $\$$ or xenon $\$$ )).ti. (63312)

14 - (intensive* or critical\$).ja,jn,jx. or (sedat\$.tw,kw. and (exp *artificial ventilation/ or (((ventilat\$ or respirat\$) adj2 (artificial\$ or mechanical\$ or pneumon $\$$ or controlled)) or (ventilat $\$$ adj3 patient\$)).tw,kw.)) (263305)

$15-13$ and $14(726)$

$16-8$ or 12 or $15(3685)$

17 - (exp animal/ or exp juvenile animal/ or adult animal/ or animal cell/ or animal tissue/ or nonhuman/ or animal experiment/ or animal model/) not human/ (7509248)

18 - (animal or animals or bitch or canine* or cat or cats or dog or dogs or feline or hamster ${ }^{*}$ or lamb or lambs or mice or monkey or monkeys or mouse or murine or pig or pigs or piglet ${ }^{*}$ or porcine or primate* or rabbit ${ }^{*}$ or rats or rat or rodent $^{*}$ or sheep* ${ }^{*}$ or veterina*).ti,kw,dq,jx. not (human* or patient ${ }^{*}$ ).mp. (2479145)

Figure 1 Embase database search strategy.

selection process. Data extraction for the included studies will be housed on a separate cloud based database document.

\section{Study selection}

The aforementioned literature search will generate a query of all potential candidate papers. Seven reviewers: $\mathrm{KG}, \mathrm{AJ}, \mathrm{DW}, \mathrm{MS}, \mathrm{CF}, \mathrm{SC}$ and $\mathrm{KJ}$ will independently review the title and abstract of these studies using the inclusion/ exclusion criteria as the first tier of review/screening. All studies will be reviewed by two independent reviewers. Conflicts between reviewers will be adjudicated by a third reviewer. Full text editions will be obtained for all selected studies. Full-text articles will be reviewed by two independent reviewers from the previous list. Reason for study exclusion will be stated. Any identified conflicts will be mediated by a third reviewer, not initially involved in advancing the study. Screening procedures will be presented using a PRISMA flow diagram.

\section{Data extraction}

Data extraction will be performed independently by all listed reviewers. Data will be extracted using a novel, standardised extraction tool. A separate template will be used for status asthmaticus, status epilepticus and difficult sedation scenarios. Each template will collect adult and paediatric data, yet discriminate between these two subgroups for more granular analysis. All extracted data will be reanalysed by a second independent reviewer. Any disagreements during data extraction will be resolved by discussion between the two reviewers, or involvement of a third reviewer if no consensus is reached.

Data extraction will include the following information: 
- General characteristics-Title, first author, country, month/year of publication, study design, study size and type of hospital.

- Clinical characteristics-Age, sex, comorbidity index/ severity of illness score, inhaled volatile agent used, concentration (end-tidal gas concentration or MAC), total duration of ventilation (hours), postvolatile ICU time, ICU mortality and LOS.

- Efficacy-(1) For all three scenarios-Timing of inhaled volatile implementation-time to initiate and duration of therapy, medical therapies/adjuncts commenced prior to inhaled volatile use, de-escalation of medical therapies during volatile use, (2) Status asthmaticus-Clinical improvement (eg, breaking of bronchospasm (ie. decreased wheeze on auscultation, improved air entry on clinical examination and/or features of improved compliance with ventilation), haemodynamic improvement), improvement in oxygenation and ventilation based on blood gases and pulse oximetry, improvement in ventilator pressures (3) Status epilepticus-Clinical improvement (eg, termination in clinical seizure activity), EEG burst suppression and/or improvement in epileptiform activity (4) difficult sedation-Improvement in sedation scores and down-titration of sedation adjuncts.

- Safety-For all three scenarios:

- Cardiovascular-arrhythmias, haemodynamics.

- Respiratory-pneumothorax, SpO2 (oxygen saturation), gas exchange.

- Neurological—seizures, tremors, delirium, other.

- Hepatorenal-volatile-induced hepatitis, AKI, fluoride nephrotoxicity, other.

- Other adverse effects in ICU (haematological, etc).

- Long-term complications.

Feasibility—For all three scenarios:

- Method of delivering inhaled volatile delivery and any technical problems (ie, miniature vaporiser, anaesthesia workstation, etc.).

- Specific personnel used to deliver inhaled volatile agents.

- Attitudes towards implementation.

- Existence of institutional protocol for inhalational anaesthetic use in the ICU.

- Additional equipment required.

\section{Assessment of risk of bias in included studies}

We expect the overall quality of evidence to be low as per Grading of Recommendations, Assessment, Development and Evaluations criteria, given this review will largely capture case reports and case series. ${ }^{22}$ However, we will use the Cochrane Risk of Bias tool for randomised controlled trials and Newcastle-Ottawa Quality Assessment Scale (NOS) for non-randomised studies including retrospective cohort, cohort and case control studies. The NOS is a three-dimensional appraisal tool to assess the methodological quality that includes (1) selected population, (2) comparability of groups and (3) outcome of interest. The study team will also use the Joanna Briggs Institute critical appraisal tools-developed according to CARE Guidelines (for CAse REports), for case reports and case series, which seeks to methodological assess the quality of a study and to determine the extent to which a study has addressed the possibility of bias in its design, conduct and analysis. ${ }^{23}{ }^{24}$ Considering the scarcity of information around this topic, no studies will be excluded based on the assessment of risk of bias. Assessment of the risk of bias will be used to illuminate the quality of the available evidence rather than explicitly weigh different papers against one another in the synthesis of data.

\section{Data synthesis and analysis}

We anticipate this systematic review to be largely based on case reports and case series. Using a narrative description, we will summarise the above outcomes separately for the three medical scenarios. Where feasible, we will summarise outcomes using tables, and collate data using descriptive statistics. Categorical variables (eg, sex, type of inhalational agent used) will be summarised using frequency (percentage), and continuous data (eg, age, duration of inhaled volatile use) will be summarised using median (IQR and/or range). If there is a sufficient number of randomised controlled trials, we will pool this information into a meta-analysis. A meta-analysis will measure the mean difference $(95 \%$ CI $)$ for continuous outcomes, and OR (95\% CI) for dichotomous outcomes. We will evaluate heterogeneity using the $\mathrm{I}^{2}$ statistic $\left(\mathrm{I}^{2}\right.$ value of more than $50 \%$ is considered moderate heterogeneity) and forest plot for randomised trials.

\section{Missing data}

If data points are missing, we will exclude individual datum while reporting the remaining available data. If a full text paper reporting a randomised trial has data presented as median and IQR we will impute the mean from the median and calculate the SD from other parameters such as the SE or the IQR. We will report missing data within the final review, but no imputation technique will be performed to account for missing information. Given the large scope of this review, we will not contact authors for missing data.

\section{Subgroup analysis and investigation of heterogeneity}

A priori subgroup analyses include (1) assessment of adult and paediatric patients, (2) volatile inhalational agents that are most commonly used, (3) duration of inhalational agent therapy and (4) method of delivery of volatile inhalational agents.

\section{Sensitivity analysis}

Most studies are expected to be of moderate to low quality, as there is a scarcity of clinical trials conducted in this area. No further sensitivity analysis based on trial quality will be performed.

\section{Patient and public involvement}

There will be no patient or public consultation process. 
Author affiliations

${ }^{1}$ Department of Anesthesiology and Pain Medicine, University of Toronto, Toronto, Ontario, Canada

${ }^{2}$ Department of Medicine, University of Western Ontario, London, Ontario, Canada

${ }^{3}$ Department of Medical Biophysics, University of Western Ontario, London, Ontario, Canada

${ }^{4}$ University of Toronto Faculty of Medicine, Toronto, Ontario, Canada

${ }^{5}$ Department of Anesthesiology, McGill University Faculty of Medicine, Montreal, Quebec, Canada

${ }^{6}$ Department of Pediatrics, Laval University Faculty of Medicine, Quebec, Canada

${ }^{7}$ The Brain Institute, Western University, London, Ontario, Canada

${ }^{8}$ Department of Anesthesia, Sunnybrook Health Sciences Centre, Toronto, Ontario, Canada

\section{Twitter Conall Francoeur@Con_All}

Contributors All authors were involved in the drafting of this manuscript. KG designed the data extraction tool. JC primarily developed the literature search strategy. KG, AJ, SC, CF, KJ, MS and DW all contributed by helping to design and write the protocol.

Funding $\mathrm{AJ}$ is funded by Merit Award, Department of Anesthesiology and Pain Medicine, University of Toronto, and Canadian Institute for Health Research.

Competing interests None declared.

Patient consent for publication Not applicable.

Provenance and peer review Not commissioned; externally peer reviewed.

Supplemental material This content has been supplied by the author(s). It has not been vetted by BMJ Publishing Group Limited (BMJ) and may not have been peer-reviewed. Any opinions or recommendations discussed are solely those of the author(s) and are not endorsed by BMJ. BMJ disclaims all liability and responsibility arising from any reliance placed on the content. Where the content includes any translated material, BMJ does not warrant the accuracy and reliability of the translations (including but not limited to local regulations, clinical guidelines, terminology, drug names and drug dosages), and is not responsible for any error and/or omissions arising from translation and adaptation or otherwise.

Open access This is an open access article distributed in accordance with the Creative Commons Attribution Non Commercial (CC BY-NC 4.0) license, which permits others to distribute, remix, adapt, build upon this work non-commercially, and license their derivative works on different terms, provided the original work is properly cited, appropriate credit is given, any changes made indicated, and the use is non-commercial. See: http://creativecommons.org/licenses/by-nc/4.0/.

\section{ORCID iDs}

Kevin Gorsky http://orcid.org/0000-0002-8480-6516

Sean Cuninghame http://orcid.org/0000-0002-2594-6121

\section{REFERENCES}

1 Hudson AE, Hemmings HC. 3 - Pharmacokinetics of inhaled anesthetics. In: Hemmings HC, Egan TD, eds. Pharmacology and Physiology for Anesthesia. Second Edition. Elsevier, 2019: 44-69.

2 Jerath A, Parotto M W, et al. Volatile anesthetics Is a new player emerging in critical care sedation?. Am J Respir Crit Care Med 2016;193:1202-12.
3 Wąsowicz M, Jerath A. [Expanding the use of volatile anesthetic agents beyond the operating room]. Can J Anaesth 2014;61:905-8.

4 Jerath A, Ferguson ND, Steel A, et al. The use of volatile anesthetic agents for long-term critical care sedation (VALTS): study protocol for a pilot randomized controlled trial. Trials 2015;16:560.

5 Mondoñedo JR, McNeil JS, Amin SD, et al. Volatile anesthetics and the treatment of severe bronchospasm: a concept of targeted delivery. Drug Discov Today Dis Models 2015;15:43-50.

6 Chakraborty RK, Basnet S. Status asthmaticus. In: StatPearls. StatPearls Publishing, 2020. http://www.ncbi.nlm.nih.gov/books/ NBK526070/

7 Wylie T, Sandhu DS, Goyal A. Status epilepticus. StatPearls Publishing, 2020. https://www.ncbi.nlm.nih.gov/books/NBK430686/

8 Zeiler FA, Zeiler KJ, Teitelbaum J, et al. Modern inhalational anesthetics for refractory status epilepticus. Can J Neurol Sci 2015;42:106-15.

9 Feng H-J, Mathews GC, Kao C, et al. Alterations of GABA A-receptor function and allosteric modulation during development of status epilepticus. J Neurophysiol 2008;99:1285-93.

10 Deeb TZ, Maguire J, Moss SJ. Possible alterations in GABAA receptor signaling that underlie benzodiazepine-resistant seizures. Epilepsia 2012;53 Suppl 9:79-88.

11 Mirsattari SM, Sharpe MD, Young GB. Treatment of refractory status epilepticus with inhalational anesthetic agents isoflurane and desflurane. Arch Neurol 2004;61:1254-9.

12 Mayer SA, Claassen J, Lokin J, et al. Refractory status epilepticus: frequency, risk factors, and impact on outcome. Arch Neurol 2002;59:205-10.

13 Shorvon S. Status epilepticus: its clinical features and treatment in children and adults. 1st edn. Cambridge University Press, 2006.

14 Claassen J, Hirsch LJ, Emerson RG, et al. Continuous EEG monitoring and midazolam infusion for refractory nonconvulsive status epilepticus. Neurology 2001;57:1036-42.

15 Franks NP, Lieb WR. Molecular and cellular mechanisms of general anaesthesia. Nature 1994;367:607-14.

16 Ries CR, Puil E. Mechanism of anesthesia revealed by shunting actions of isoflurane on thalamocortical neurons. J Neurophysiol 1999;81:1795-801.

17 Mehta S, Burry L, Fischer S, et al. Canadian survey of the use of sedatives, analgesics, and neuromuscular blocking agents in critically ill patients. Crit Care Med 2006;34:374-80.

18 Payen J-F, Chanques G, Mantz J, et al. Current practices in sedation and analgesia for mechanically ventilated critically ill patients: a prospective multicenter patient-based study. Anesthesiology 2007:106:687-95

19 Jerath A, Beattie SW, Chandy T, et al. Volatile-based short-term sedation in cardiac surgical patients: a prospective randomized controlled trial. Crit Care Med 2015;43:1062-9.

20 Landoni G, Saleh O, Scarparo E. Volatile anesthetics for intensive care unit sedation. In: De Gaudio AR, Romagnoli S, eds. Critical Care Sedation. Springer International Publishing, 2018: 103-19.

21 Flippo TS, Holder WD. Neurologic degeneration associated with nitrous oxide anesthesia in patients with vitamin B12 deficiency. Arch Surg 1993;128:1391-5.

22 Guyatt GH, Oxman AD, Vist GE, et al. GRADE: an emerging consensus on rating quality of evidence and strength of recommendations. BMJ 2008;336:924-6.

23 Gagnier JJ, Kienle G, Altman DG, et al. The care guidelines: consensus-based clinical case reporting Guideline development. Headache 2013;53:1541-7.

24 Martin J. () Joanna Briggs Institute 2017 critical appraisal checklist for case reports. 5, 2017. 